\title{
Research Square \\ Kinematic Calibration of a 7R 6-DOF Robot With Non-spherical Wrist Using Laser Tracker
}

\section{Xuhao Wang ( $\nabla$ xh_wang@cauc.edu.cn )}

Aeronautical Engineering College, Civil Aviation University of China, Tianjin, 300300, China https://orcid.org/0000-0003-3042-9679

\section{Dawei Zhang}

Tianjin University

\section{Chen Zhao}

Tianjin University

\section{Zhiyong Guo}

Civil Aviation University of China

\section{Mengli Wu}

Civil Aviation University of China

\section{Xiangzhi Meng}

Automotive Engineering Corporation

\section{Li Ma}

Automotive Engineering Corporation

\section{Original Article}

Keywords: Robot calibration, Kinematic identification, Parameter identifiability, Mode reduction, 7R 6-DOF robot

Posted Date: August 21st, 2020

DOl: https://doi.org/10.21203/rs.3.rs-60243/v1

License: (1) (1) This work is licensed under a Creative Commons Attribution 4.0 International License.

Read Full License 


\title{
Kinematic calibration of a 7R 6-DOF robot with non-spherical wrist using laser tracker
}

\author{
Xuhao Wang ${ }^{1, *}$, Dawei Zhang ${ }^{2}$, Chen Zhao ${ }^{2, *}$, Zhiyong Guo ${ }^{1}$, Mengli Wu ${ }^{1}$, Xiangzhi Meng ${ }^{3}$, Li Ma $^{3}$ \\ 1 Aeronautical Engineering College, Civil Aviation University of China, Tianjin, 300300, China \\ 2 Key Laboratory of Mechanism Theory and Equipment Design of Ministry of Education, School of Mechanical \\ Engineering, Tianjin University, Tianjin, 300350, China \\ 3 Automotive Engineering Corporation, Tianjin, 300113, China
}

\begin{abstract}
In this paper, a kinematic calibration method is proposed to improve the accuracy of a 7R 6-DOF robot with non-spherical wrist, where the parameters identifiability analysis and model reduction are particularly addressed in the identification procedure. Firstly, the kinematic error model of the robot is constructed based on the modified DH (MDH) model. Secondly, in order to derive a minimal model which is more suitable for practical application, model reduction is implemented considering compensability and constraints of the parameters defined in the controller, and QR decomposition is utilized to determine the redundant parameters, which are then removed in practical parameter identification. Finally, by using a laser tracker as the measuring device, a two-step procedure for error parameter estimation is proposed: (1) estimation of the robot base location with respect to the measurement coordinate system; (2) fine estimation of the identifiable kinematic parameters according to the measurement data. The experiment on a 7R 6-DOF industrial painting robot is implemented, the results show that the proposed method can effectively improve the accuracy of the robot.
\end{abstract}

Keywords: Robot calibration; Kinematic identification; Parameter identifiability; Mode reduction; 7R 6-DOF robot.

\section{Introduction}

Repeatability and accuracy are most crucial performance indices of industrial robots. Especially, with the expansion of robots' application area and off-line programming, accuracy is more and more essential. It is well known that if the robot has sufficient repeatability, calibration is an efficient way to improve robot accuracy $[1,2]$. In fact, there are many factors could influence the positioning accuracy of industrial robots, e.g. geometric parameter errors, flexible deformation, thermal distortion and expansion and so on. However, as reported by several references $[3,4]$, the kinematic errors contribute for more than $90 \%$ of the total errors. Therefore, this paper mainly focuses on kinematic calibration of a 7R 6-DOF painting robot with non-spherical wrist.

The robot calibration procedure generally involves four steps [5]: modeling, measurement, identification, and compensation. The modeling and identification issues would be particularly addressed in this paper. The modeling step is to construct a mathematical relationship between the kinematic errors and the pose of the end-effector (EE), and it is the premise and foundation of calibration. Schröer [6] pointed out that a perfect model should satisfy three conditions: continuousness, completeness and minimality. To date, the Denavit-Hartenberg (DH) model [7] is universally utilized for robot kinematic calibration $[8,9]$. However, DH model is discontinuous when two consecutive joint axes are parallel or near parallel. To solve this problem, Hayati [10] firstly modified this model by adding an extra revolute parameter around the $\mathrm{Y}$ axis i.e. the modified $\mathrm{DH}(\mathrm{MDH})$ model. Then, authors have presented some other modified models to guarantee continuousness condition, e.g. S model [11], Zero-reference model [12], CPC model [13], and so on. The POE-based model is another competitive choice in the robot calibration $[14,15]$, because it internally satisfies the continuousness and completeness conditions. However, a potential drawback of these alternate modeling methods is that the compensation cannot be directly implementable in controllers, because DH notation still dominates the kinematic models used in the controller of existing actual industrial manipulators. It should be noted that the kinematic model is generally redundant, in other words, some parameters are not identifiable especially for the modified models with additional parameters to ensure continuousness. This leads to the parameter identifiability analysis and model reduction problem, and there have been several techniques for elimination of these redundant parameters. For example, Khalil and Dombre [16] used QR decomposition of the identification Jacobian for model redundancy analysis, while Gao [17] utilized singular value decomposition (SVD) to solve this problem. Additionally, some of the kinematic parameters cannot be directly changed in controller, because the inverse kinematics may be based on the properties of these parameters e.g. $\alpha_{i}$ equals $\pm 90^{\circ}$ or $0^{\circ}$. Therefore, model parameter reduction considering the compensability and constraints defined in the robot controller is still an open issue needs to be further investigated.

As for measurement step, there are mainly two techniques: the closed-loop and open-loop ones. The closed-loop method applies constrains (e.g. point, plane or sphere constraints) on the EE of the robot and collects joint angles by encoders $[18,19]$. It does not rely on any expensive measurement device, however the robot must be carefully operated to satisfy particular constrains which requires skilled personnel and

${ }^{*}$ Corresponding author, Tel./fax:+86 22 24092418, E-mail: xh_wang@cauc.edu.cn (X. Wang) Tel./fax:+86 22 27406951, E-mail: zhaochen@tju.edu.cn (C. Zhao) 
is time consuming. Therefore, the open-loop methods with external measurement devices e.g. laser tracker [5, 20], measurement arms [15, 21], ball-bar system [22, 23], wire potentiometer [8], photogrammetry system $[24,25]$, and so on, to measure full or partial pose of the EE have been widely used. In this case, the measured data is described in the measurement coordinate system. So, the estimation of robot base location with respect to the measurement coordinate system is an essential work before kinematic parameter identification, which generally needs additional measurement. Besides, some authors have proposed techniques to improve the parameter identification accuracy by optimal selection of measurement configurations for calibration experiments [26, 27].

The robot researched in this paper is a 7R 6-DOF robot as shown in Figure 1, which is not a purely open-loop structure, but rather contains a 4R 3-DOF non-spherical wrist with a coupling joint to increases motion flexibility [28]. This special wrist great increase the complexity and difficulty of calibration. To develop a feasible kinematic calibration method more suitable for industrial application, kinematic error model of the robot is constructed based on the modified DH (MDH) model. Then, model reduction and redundancy analysis are implemented considering compensability and constraints of the parameters defined in the controller. Finally, special attention is applied on estimation of the robot base location with respect to the measurement coordinate system before fine identification of the kinematic parameters. Having outlined existing approaches and challenges, this paper is organized in the following manner. In Section 2, the kinematic model of the robot is constructed, and the error parameter estimation method is proposed. Section 3 gives parameters identifiability analysis and model reduction. Section 4 conducts the kinematic calibration experiment based on a 7R 6-DOF painting robot. And the conclusions are drawn in Section 5.

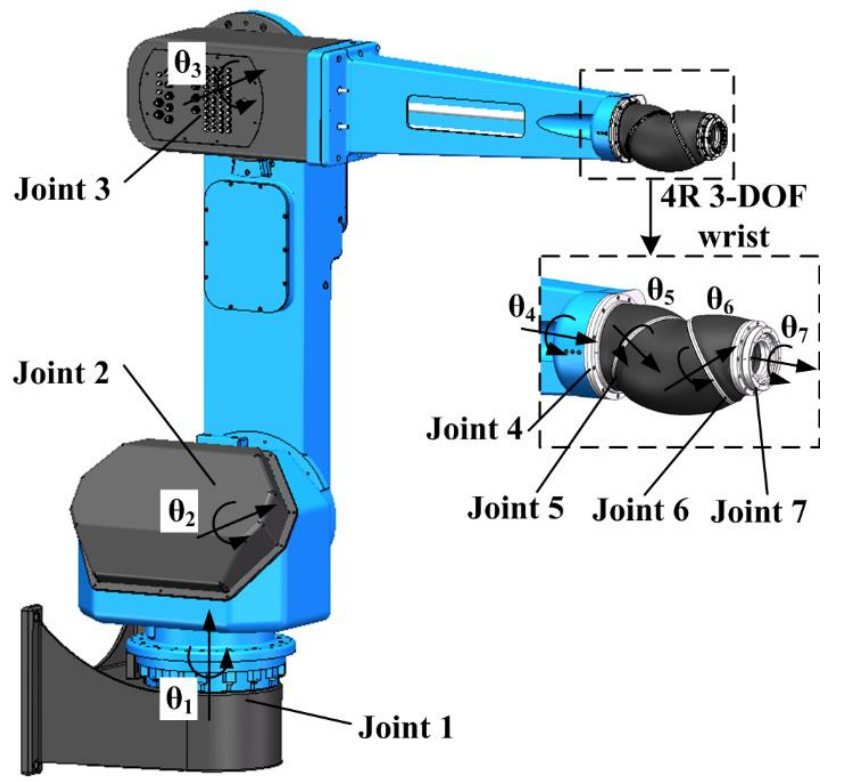

(a) Virtual prototype

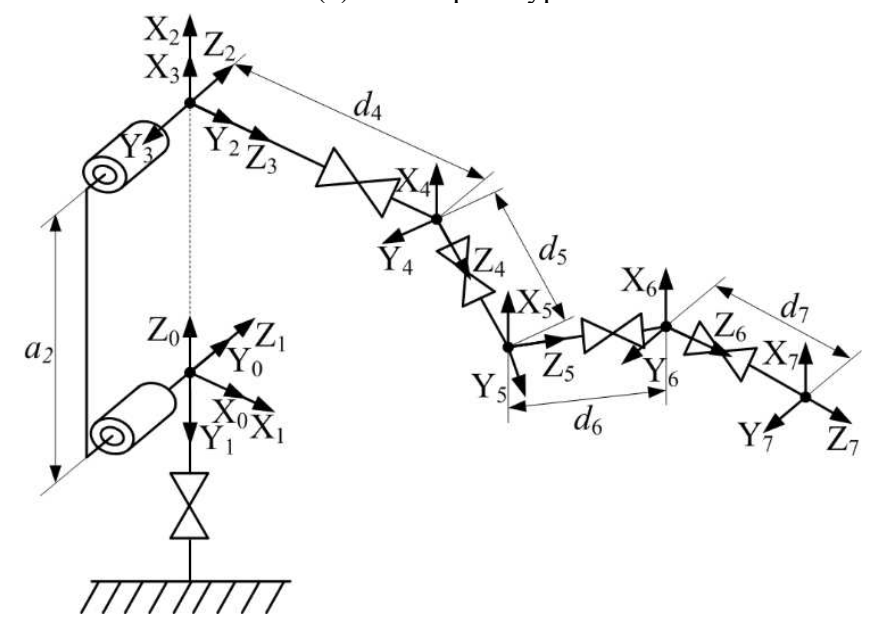

(b) Schematic diagram and coordinate systems

Figure 1 The 7R 6-DOF painting robot with non-spherical wrist (a) Virtual prototype (b) Schematic diagram and coordinate systems 


\section{Modeling and parameter identification}

\subsection{Kinematic parameter error modeling}

The kinematic error model is a mathematical relationship between the kinematic errors and the pose of EE. As shown in Figure 1 (a), it is the virtual prototype of a 7R 6-DOF robot with non-spherical wrist which has been proved to be advantageous in painting application due to wider range of motion of the wrist [28]. The 4R 3-DOF wrist of the 7R 6-DOF robot has 4 revolute joints, the second and third of which are coupled with the relation $\theta_{6}=-\theta_{5}$. This paper aims at developing kinematic calibration method that is more suitable for industrial application, then the kinematic model is established based on $\mathrm{MDH}$ model considering that the second and third joint axes are parallel. Figure 1 (b) shows the coordinate systems of the 7R 6-DOF robot, while the corresponding MDH parameters are listed in Table 1. For general case, the transformation from coordinate system $\{i-1\}$ to $\{i\}$ is described by four parameters and is expressed as

$$
\begin{aligned}
{ }_{i}^{i-1} \mathbf{T} & =\operatorname{Rot}\left(z, \theta_{i}\right) \operatorname{Trans}\left(0,0, d_{i}\right) \operatorname{Rot}\left(x, \alpha_{i}\right) \operatorname{Trans}\left(a_{i}, 0,0\right) \\
& =\left[\begin{array}{cccc}
c_{i} & -s_{i} c \alpha_{i} & s_{i} s \alpha_{i} & a_{i} c_{i} \\
s_{i} & c_{i} c \alpha_{i} & -c_{i} s \alpha_{i} & a_{i} s_{i} \\
0 & s \alpha_{i} & c \alpha_{i} & d_{i} \\
0 & 0 & 0 & 1
\end{array}\right]
\end{aligned}
$$

where $s_{i}=\sin \theta_{i}, \quad c_{i}=\cos \theta_{i}, c \alpha_{i}=\cos \alpha_{i}, \quad s \alpha_{i}=\sin \alpha_{i}$.

Table 1 Nominal values of the MDH parameters of the 7R 6-DOF painting robot

\begin{tabular}{cccccc}
\hline $\boldsymbol{i}$ - th & $\theta_{i}(\mathrm{deg})$ & $d_{i}(\mathrm{~mm})$ & $a_{i}(\mathrm{~mm})$ & $\alpha_{i}(\mathrm{deg})$ & $\beta_{i}(\mathrm{deg})$ \\
\hline 1 & $\theta_{1}$ & 0 & 0 & -90 & $/$ \\
2 & $\theta_{2}-90$ & 0 & 1300 & 0 & 0 \\
3 & $\theta_{3}$ & 0 & 0 & -90 & $/$ \\
4 & $\theta_{4}$ & 1474 & 0 & -35 & $/$ \\
5 & $\theta_{5}$ & 95 & 0 & 70 & $/$ \\
6 & $\theta_{6}$ & 95 & 0 & -35 & $/$ \\
7 & $\theta_{7}$ & 85 & 0 & 0 & 0 \\
\hline
\end{tabular}

However, when two consecutive joint axes are parallel, an extra revolute parameter around the $\mathrm{Y}$ axis is added, the corresponding transformation matrix can be expressed as

$$
\begin{aligned}
{ }_{i}^{i-1} \mathbf{T} & =\operatorname{Rot}\left(z, \theta_{i}\right) \operatorname{Trans}\left(0,0, d_{i}\right) \operatorname{Rot}\left(x, \alpha_{i}\right) \operatorname{Trans}\left(a_{i}, 0,0\right) \operatorname{Rot}\left(y, \beta_{i}\right) \\
& =\left[\begin{array}{cccc}
c_{i} c \beta_{i}-s_{i} c \alpha_{i} s \beta_{i} & -s_{i} c \alpha_{i} & c_{i} s \beta_{i}+s_{i} s \alpha_{i} c \beta_{i} & a_{i} c_{i} \\
s_{i} c \beta_{i}+c_{i} s \alpha_{i} s \beta_{i} & c_{i} c \alpha_{i} & s_{i} s \beta_{i}-c_{i} s \alpha_{i} c \beta_{i} & a_{i} s_{i} \\
-c \alpha_{i} s \beta_{i} & s \alpha_{i} & c \alpha_{i} c \beta_{i} & d_{i} \\
0 & 0 & 0 & 1
\end{array}\right]
\end{aligned}
$$

where $s \beta_{i}=\sin \left(\beta_{i}\right), c \beta_{i}=\cos \left(\beta_{i}\right)$. Then, forward kinematics of the robot i.e. the transformation matrix from the world reference frame to its $\mathrm{EE}$ frame could be formulated by

$$
{ }_{\text {tool }}^{\text {base }} \mathbf{T}=\mathbf{T}_{\text {base }}{ }_{1}^{0} \mathbf{T}_{2}^{1} \mathbf{T}_{3}^{2} \mathbf{T}_{4}^{3} \mathbf{T}_{5}^{4} \mathbf{T}_{6}^{5} \mathbf{T}_{7}^{6} \mathbf{T} \mathbf{T}_{\text {tool }}=\mathbf{T}_{\text {base }} \prod_{i=1}^{7}{ }_{i}^{i-1} \mathbf{T} \mathbf{T}_{\text {tool }}
$$

where $\mathbf{T}_{\text {base }}$ is the transformation matrix from the world frame to base frame, $\mathbf{T}_{\text {tool }}$ is the transformation matrix from frame $\{7\}$ to EE frame. These transformation matrices are described by six parameters and formulated as Eqs. (4) and (5). 


$$
\begin{aligned}
\mathbf{T}_{\text {base }} & =\operatorname{Trans}\left(x_{0}, y_{0}, z_{0}\right) \operatorname{Rot}\left(x, \alpha_{0}\right) \operatorname{Rot}\left(y, \beta_{0}\right) \operatorname{Rot}\left(z, \varphi_{0}\right) \\
& =\left[\begin{array}{cccc}
c \beta_{0} c \varphi_{0} & -c \beta_{0} s \varphi_{0} & s \beta_{0} & x_{0} \\
c \alpha_{0} s \varphi_{0}+s \alpha_{0} s \beta_{0} c \varphi_{0} & c \alpha_{0} c \varphi_{0}-s \alpha_{0} s \beta_{0} s \varphi_{0} & -s \alpha_{0} c \beta_{0} & y_{0} \\
s \alpha_{0} s \varphi_{0}-c \alpha_{0} s \beta_{0} c \varphi_{0} & s \alpha_{0} c \varphi_{0}+c \alpha_{0} s \beta_{0} s \varphi_{0} & c \alpha_{0} c \beta_{0} & z_{0} \\
0 & 0 & 0 & 1
\end{array}\right] \\
\mathbf{T}_{\text {tool }} & =\operatorname{Trans}\left(x_{t}, y_{t}, z_{t}\right) \operatorname{Rot}\left(x, \alpha_{t}\right) \operatorname{Rot}\left(y, \beta_{t}\right) \operatorname{Rot}\left(z, \varphi_{t}\right) \\
& =\left[\begin{array}{cccc}
c \beta_{t} c \varphi_{t} & -c \beta_{t} s \varphi_{t} & s \beta_{t} & x_{t} \\
c \alpha_{t} s \varphi_{t}+s \alpha_{t} s \beta_{t} c \varphi_{t} & c \alpha_{t} c \varphi_{t}-s \alpha_{t} s \beta_{t} s \varphi_{t} & -s \alpha_{t} c \beta_{t} & y_{t} \\
s \alpha_{t} s \varphi_{t}-c \alpha_{t} s \beta_{t} c \varphi_{t} & s \alpha_{t} c \varphi_{t}+c \alpha_{t} s \beta_{t} s \varphi_{t} & c \alpha_{t} c \beta_{t} & z_{t} \\
0 & 0 & 0 & 1
\end{array}\right]
\end{aligned}
$$

where $\left[x_{0}, y_{0}, z_{0}\right]\left(\left[x_{t}, y_{t}, z_{t}\right]\right)$ is the translation vector from the world frame to base frame (from frame $\{7\}$ to $\mathrm{EE}$ frame), $\left[\alpha_{0}, \beta_{0}, \varphi_{0}\right]\left(\left[\alpha_{t}, \beta_{t}, \varphi_{t}\right]\right)$ is the corresponding Euler rotation angles vector.

In order to construct the kinematic error model, the errors of kinematic parameters are defined as $\delta \mathbf{q}_{i}=\left[\Delta \theta_{i}, \Delta d_{i}, \Delta \alpha_{i}, \Delta a_{i}, \Delta \beta_{i}\right]^{\mathrm{T}}(i=1, \cdots, 7), \delta \mathbf{q}_{k}=\left[\Delta x_{k}, \Delta y_{k}, \Delta z_{k}, \Delta \alpha_{k}, \Delta \beta_{k}, \Delta \varphi_{k}\right]^{\mathrm{T}}(k=0, t)$. Then, the actual configuration of $\mathrm{EE}$ with respect to the world frame can be rewritten as

$$
{ }_{\text {bosel }}^{\text {base }} \mathbf{T}^{r}=\mathbf{T}_{\text {base }}^{r} \prod_{i=1}^{7}{ }_{i}^{i-1} \mathbf{T}^{r} \mathbf{T}_{\text {tool }}^{r}=\mathbf{T}_{\text {base }}^{r}\left(\delta \mathbf{q}_{0}\right) \prod_{i=1}^{7}{ }_{i}^{i-1} \mathbf{T}^{r}\left(\theta_{i}, \delta \mathbf{q}_{i}\right) \mathbf{T}_{\text {tool }}^{r}\left(\delta \mathbf{q}_{t}\right)
$$

where the superscripts " $r$ " represents the actual transformation. From Eqs. (3) and (6), the EE transformation matrix errors result from the errors of link, base and tool transformation matrices. The link transformation matrix error can be expressed as

$$
d_{i}^{i-1} \mathbf{T}={ }_{i}^{i-1} \mathbf{T}^{r}\left(\theta_{i}, \delta \mathbf{q}_{i}\right)-{ }_{i}^{i-1} \mathbf{T}
$$

The actual link transformation matrix can be treated as the result of the normal transformation after serial differential motions, which can be written as

$$
{ }_{i}^{i-1} \mathbf{T}^{r}\left(\theta_{i}, \delta \mathbf{q}_{i}\right)={ }_{i}^{i-1} \mathbf{T} \operatorname{Trans}\left(\delta x_{i}, \delta y_{i}, \delta z_{i}\right) \operatorname{Rot}\left(x, \delta \alpha_{i}\right) \operatorname{Rot}\left(y, \delta \beta_{i}\right) \operatorname{Rot}\left(z, \delta \varphi_{i}\right)
$$

where $\mathbf{e}_{i}=\left[\delta \mathrm{x}_{i}, \delta \mathrm{y}_{i}, \delta \mathrm{z}_{i}, \delta \alpha_{i}, \delta \beta_{i}, \delta \varphi_{i}\right]^{\mathrm{T}}$ is the link differential error vector. From Eqs. (7) and (8), following equation can be obtained

$$
\begin{aligned}
d_{i}^{i-1} \mathbf{T} & ={ }_{i}^{i-1} \mathbf{T}\left(\operatorname{Trans}\left(\delta x_{i}, \delta y_{i}, \delta z_{i}\right) \operatorname{Rot}\left(x, \delta \alpha_{i}\right) \operatorname{Rot}\left(y, \delta \beta_{i}\right) \operatorname{Rot}\left(z, \delta \varphi_{i}\right)-\mathbf{I}\right) \\
& ={ }_{i}^{i-1} \mathbf{T} \boldsymbol{\Delta}_{i}
\end{aligned}
$$

where $\boldsymbol{\Delta}_{i}=\operatorname{Trans}\left(\delta x_{i}, \delta y_{i}, \delta z_{i}\right) \operatorname{Rot}\left(x, \delta \alpha_{i}\right) \operatorname{Rot}\left(y, \delta \beta_{i}\right) \operatorname{Rot}\left(z, \delta \varphi_{i}\right)-\mathbf{I}$ is the differential operator. By ignoring the second and higher order error terms, $\boldsymbol{\Delta}_{i}$ can be expressed as

$$
\boldsymbol{\Delta}_{i}=\left[\begin{array}{cccc}
0 & -\delta \varphi_{i} & \delta \beta_{i} & \delta x_{i} \\
\delta \varphi_{i} & 0 & -\delta \alpha_{i} & \delta y_{i} \\
-\delta \beta_{i} & \delta \alpha_{i} & 0 & \delta z_{i} \\
0 & 0 & 0 & 0
\end{array}\right]
$$

From Eqs. (9) and (10), ignoring the second and higher order error terms, $\mathbf{e}_{i}$ can be written as function of the link kinematic errors.

$$
\mathbf{e}_{i}=\left[\begin{array}{c}
f_{x}\left(\Delta \theta_{i}, \Delta d_{i}, \Delta \alpha_{i}, \Delta a_{i}, \Delta \beta_{i}\right) \\
f_{y}\left(\Delta \theta_{i}, \Delta d_{i}, \Delta \alpha_{i}, \Delta a_{i}, \Delta \beta_{i}\right) \\
\ldots \\
f_{\varphi}\left(\Delta \theta_{i}, \Delta d_{i}, \Delta \alpha_{i}, \Delta a_{i}, \Delta \beta_{i}\right)
\end{array}\right]=\mathbf{M}_{i} \delta \mathbf{q}_{i}
$$

where $\mathbf{M}_{i} \in R^{6 \times 5}$ is the transform matrix between $\mathbf{e}_{i}$ and $\delta \mathbf{q}_{i}$. Similarly, the base (tool) differential error vector can be written as function of corresponding kinematic errors. See Appendix A for details of these transformation matrices. 
$\mathbf{e}_{0}=\mathbf{M}_{0} \delta \mathbf{q}_{0}, \mathbf{e}_{t}=\mathbf{M}_{t} \delta \mathbf{q}_{t}$

According to the differential theory and linear superposition principle, the total deflections of EE can be obtained with the general Jacobian matrix.

$$
\mathbf{E}^{k}=\sum_{i=1}^{7}{ }_{i}^{t} \mathbf{S e}_{i}+{ }_{0}^{t} \mathbf{S e}_{0}+{ }_{t}^{t} \mathbf{S e}_{t}
$$

where ${ }_{i}^{t} \mathbf{S}$ is the general Jacobian matrix [29], and ${ }_{t}^{t} \mathbf{S}=\mathbf{I}$. By substituting Eqs. (11) and (12) into Eq. (13), the kinematic error model can be expressed as

$$
\mathbf{E}^{k}=\sum_{i=1}^{7}{ }_{i}^{t} \mathbf{S} \mathbf{M}_{i} \delta \mathbf{q}_{i}+{ }_{0}^{t} \mathbf{S} \mathbf{M}_{0} \delta \mathbf{q}_{0}+{ }_{t}^{t} \mathbf{S} \mathbf{M}_{t} \delta \mathbf{q}_{t}=\mathbf{H}^{k} \delta \mathbf{q}
$$

where $\mathbf{E}^{k}=\left[\mathbf{E}_{p}^{k} ; \mathbf{E}_{r}^{k}\right] \in R^{6}$ is the differential error vector of EE, while $\mathbf{E}_{p}^{k}, \mathbf{E}_{r}^{k}$ denote respectively the position error vector and orientation error vector, $\delta \mathbf{q}=\left[\delta \mathbf{q}_{1}, \delta \mathbf{q}_{2}, \cdots \delta \mathbf{q}_{7}, \delta \mathbf{q}_{0}, \delta \mathbf{q}_{t}\right]^{T} \in R^{c}$ is the error vector of the kinematic parameters, $\mathbf{H}^{k}=\left[\mathbf{G}_{1} \mathbf{M}_{1}, \mathbf{G}_{2} \mathbf{M}_{2}, \cdots \mathbf{G}_{7} \mathbf{M}_{7}, \mathbf{G}_{0} \mathbf{M}_{0}, \mathbf{G}_{\mathbf{t}} \mathbf{M}_{t}\right]$ is the identification Jacobian. It is worthwhile to point out that the effects of the coupled joint of the 4R 3-DOF wrist is not considered in the identification model i.e. Eq. (14). To construct the identification model of the 7R 6DOF robot, effects of the coupled joint i.e. $\theta_{6}=-\theta_{5}$ should be analyzed. It means the joint angle error of joint $5 \Delta \theta_{5}$ could result in an additional error on joint angle of joint 6 i.e. $\Delta \theta_{6, \text { add }}=-\Delta \theta_{5}$. Therefore, the actual differential error of EE results from $\Delta \theta_{5}$ can be written as

$$
\mathbf{E}_{\Delta \theta_{5}}^{k}=\mathbf{H}^{k}\left(:, \Delta \theta_{5}\right) \Delta \theta_{5}+\mathbf{H}^{k}\left(:, \Delta \theta_{6}\right) \Delta \theta_{6, \text { add }}=\left[\mathbf{H}^{k}\left(:, \Delta \theta_{5}\right)-\mathbf{H}^{k}\left(:, \Delta \theta_{6}\right)\right] \Delta \theta_{5}
$$

where $\mathbf{H}^{k}\left(:, \Delta \theta_{5}\right), \mathbf{H}^{k}\left(:, \Delta \theta_{6}\right)$ are respectively columns of the identification Jacobian corresponding to $\Delta \theta_{5}$ and $\Delta \theta_{6}$. From Eq. (15), the identification Jacobian considering effects of the coupled joint can be derived by adjusting the column corresponding to $\Delta \theta_{5}$ as

$$
\mathbf{H}^{k}\left(:, \Delta \theta_{5}\right)=\mathbf{H}^{k}\left(:, \Delta \theta_{5}\right)-\mathbf{H}^{k}\left(:, \Delta \theta_{6}\right)
$$

\subsection{Kinematic parameter identification}

In order to identify the actual kinematic parameters, we need to measure poses of $\mathrm{EE}$ in different configurations and calculate the differential errors of EE. From Eq. (14), $6 \times N$ scalar equations can be obtained as

$$
\left[\begin{array}{c}
\mathbf{E}^{1} \\
\mathbf{E}^{2} \\
\cdots \\
\mathbf{E}^{N}
\end{array}\right]=\left[\begin{array}{c}
\mathbf{H}^{1} \\
\mathbf{H}^{2} \\
\cdots \\
\mathbf{H}^{N}
\end{array}\right] \delta \mathbf{q}, \quad \mathbf{E}=\mathbf{H} \delta \mathbf{q}
$$

where $N$ is the number of measurement. When $6 \times N>c$, by applying the least-square technique, the error vector of kinematic parameters $\delta \mathbf{q}$ can be obtained as

$$
\delta \mathbf{q}=\mathbf{H}^{+} \mathbf{E}=\left(\mathbf{H}^{T} \mathbf{H}\right)^{-1} \mathbf{H}^{T} \mathbf{E}
$$

where $\mathbf{H}^{+}=\left(\mathbf{H}^{T} \mathbf{H}\right)^{-1} \mathbf{H}^{T}$ is the pseudo-inverse of $\mathbf{H}$. It is notable that the identification Jacobian $\mathbf{H}^{k}$ in Eq. (14) is function of the kinematic parameters of links, base and tool. In other words, the normal values of these parameters would be used in Eq. (18). For a specific robot, normal values of kinematic parameters of links i.e. MDH parameters are known. In addition, if manufacture errors of the measurement tool are relatively small, the normal values of tool kinematic parameters are also treated as known. Thus, only the base kinematic parameters are unknown. To solve this problem, the existing method is to do a preliminary identification of the base transformation matrix by additional measuring the axes 1 and 2 with external measurement device e.g. laser tracker $[1,30]$. In order to simplify the identification process, this paper proposed a two-step procedure for kinematic parameter estimation: (1) estimation of the robot base location with respect to the world frame, (2) fine estimation of the identifiable kinematic parameters. Because no additional measurements are needed, the proposed method is simpler and more suitable for actual industrial application.

In the first step, the kinematic parameters of links and tool transformation are treated as known and take their normal values. From Eq. (6), for each measurement, the configuration of EE with respect to the world frame can be expressed as 


$$
{ }_{\text {tool }}^{\text {base }} \mathbf{T}^{r(k)}=\mathbf{T}_{\text {base }} \prod_{i=1}^{7}{ }_{i}^{i-1} \mathbf{T}^{(k)} \mathbf{T}_{\text {tool }}=\mathbf{T}_{\text {base }} \mathbf{T}_{\text {tool }}^{0(k)}
$$

where $\mathbf{T}_{\text {tool }}^{0(k)}=\prod_{i=1}^{7}{ }_{i}^{i-1} \mathbf{T}^{(k)} \mathbf{T}_{\text {tool }}$ is the configuration of EE with respect to base frame. From Eq. (19), the coarse estimation of the base transformation can be obtained as

$$
\tilde{\mathbf{T}}_{\text {base }}={ }_{\text {bool }}^{\text {base }} \mathbf{T}^{r(k)}\left(\mathbf{T}_{\text {tool }}^{0(k)}\right)^{-1}
$$

The normal base transformation is treated as the result of coarse estimation after differential motions.

$$
\mathbf{T}_{\text {base }}=\tilde{\mathbf{T}}_{\text {base }}\left(\mathbf{I}+\boldsymbol{\Delta}_{\text {base }}\right)
$$

where $\boldsymbol{\Delta}_{\text {base }}$ is the differential operator of base frame and can be written in differential error vector form $\mathbf{e}_{\text {base }}=\left[\delta \tilde{\mathbf{d}}_{\text {base }}, \delta \tilde{\mathbf{r}}_{\text {base }}\right]^{\mathrm{T}}=\left[\delta \tilde{x}_{\text {base }}, \delta \tilde{y}_{\text {base }}, \delta \tilde{z}_{\text {base }}, \delta \tilde{\alpha}_{\text {base }}, \delta \tilde{\beta}_{\text {base }}, \delta \tilde{\varphi}_{\text {base }}\right]^{\mathrm{T}}$. Any homogeneous transformation matrix can be written by rotational submatrix $\mathbf{R}^{3 \times 3}$ and translational vector $\mathbf{P}^{3 \times 1}$ as

$$
\mathbf{T}=\left[\begin{array}{cc}
\mathbf{R}^{3 \times 3} & \mathbf{P}^{3 \times 1} \\
\mathbf{0}^{1 \times 3} & 1
\end{array}\right]
$$

From Eqs. (19) and (21), for each measurement, the position vector of EE can be expressed as

$$
\begin{aligned}
& { }_{\text {tool }}{ }^{(k)}=\tilde{\mathbf{R}}_{\text {base }}\left(\mathbf{I}+\operatorname{Sp}\left(\delta \tilde{\mathbf{r}}_{\text {base }}\right)\right) \mathbf{P}_{\text {tool }}^{0(k)}+\tilde{\mathbf{R}}_{\text {base }} \delta \tilde{\mathbf{d}}_{\text {base }}+\tilde{\mathbf{P}}_{\text {base }} \\
& =\tilde{\mathbf{R}}_{\text {base }} \mathbf{P}_{\text {tool }}^{0(k)}-\tilde{\mathbf{R}}_{\text {base }} \operatorname{Sp}\left(\mathbf{P}_{\text {tool }}^{0(k)}\right) \delta \tilde{\mathbf{r}}_{\text {base }}+\tilde{\mathbf{R}}_{\text {base }} \delta \tilde{\mathbf{d}}_{\text {base }}+\tilde{\mathbf{P}}_{\text {base }}
\end{aligned}
$$

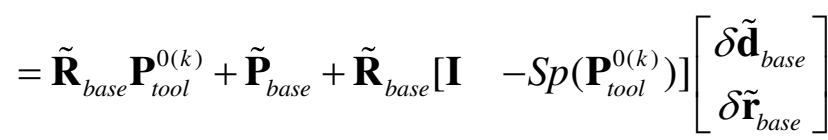

where $\operatorname{Sp}\left(\mathbf{P}_{\text {tool }}^{0(k)}\right)$ is skew symmetric matrix of $\mathbf{P}_{\text {tool }}^{0(k)}$. From Eq. (23), by applying the least-square technique, the differential error vector of base frame can be obtained as

$$
\mathbf{e}_{\text {base }}=\left[\begin{array}{c}
\delta \tilde{\mathbf{d}}_{\text {base }} \\
\delta \tilde{\mathbf{r}}_{\text {base }}
\end{array}\right]=\left(\mathbf{A}_{c}^{\mathrm{T}} \mathbf{A}_{c}\right)^{-1} \mathbf{A}_{c}^{\mathrm{T}} \Delta \mathbf{P}_{c}
$$

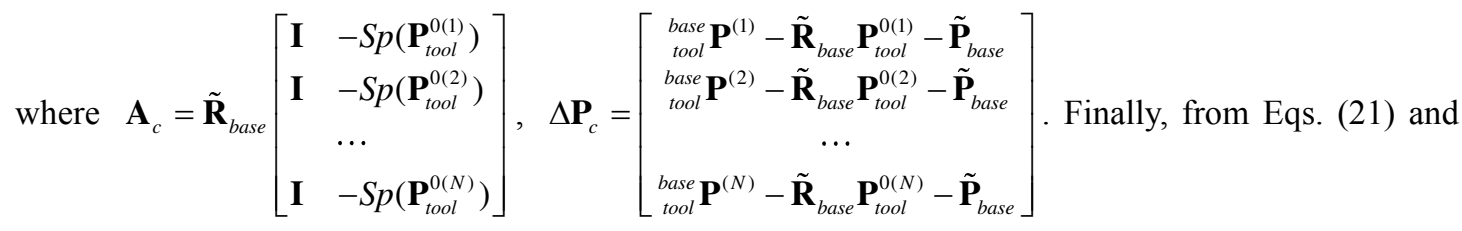

(24), the normal base transformation matrix can be derived.

In the second step, the normal kinematic parameters of links, base transformation and tool transformation are treated as known. Then from Eq. (18), the error vector of kinematic parameters can be obtained.

\section{Model reduction and parameter identifiability analysis}

The original $\mathrm{MDH}$ model satisfies the continuousness and completeness conditions, however it generally do not satisfy the minimality condition i.e. some kinematic parameters are redundant. Additionally, some kinematic parameters used in controllers cannot be directly changed, because the inverse kinematics may be based on the properties of these parameters e.g. $\alpha_{i}$ equals $\pm 90^{\circ}$ or $0^{\circ}$. Therefore, in order to develop a calibration method that is suitable for practical application, model reduction and parameters identifiability analysis should be conducted before parameter identification.

\subsection{Model reduction}

According to the compensability of the parameters in the robot controller, these parameters can be divided into two groups: G1-parameters that can be compensated by directly changing values in the controller; G2 - other parameters. It is notable that the base and tool transformation matrix can be redefined in the controller, therefore the corresponding kinematic parameters belong to G1. Detailed classification of the kinematic parameters of the 7R 6-DOF robot is listed in Table 2.

To guarantee the developed calibration method suitable for practical application, parameters in G1 are retained, while those in $\mathrm{G} 2$ are removed. By removing columns of $\mathbf{H}^{k}$ corresponding to the parameters in $\mathrm{G} 2$, the reduced model is obtained as 
Table 2 Classification of the kinematic parameters of the 7R 6-DOF robot

\begin{tabular}{cccccc}
\hline $\boldsymbol{i}$ - th & $\theta_{i}$ & $d_{i}$ & $a_{i}$ & $\alpha_{i}$ & $\beta_{i}$ \\
\hline 1 & $\mathrm{G} 1$ & $\mathrm{G} 1$ & $\mathrm{G} 1$ & $\mathrm{G} 2$ & $/$ \\
2 & $\mathrm{G} 1$ & $\mathrm{G} 1$ & $\mathrm{G} 1$ & $\mathrm{G} 2$ & $\mathrm{G} 2$ \\
3 & $\mathrm{G} 1$ & $\mathrm{G} 2$ & $\mathrm{G} 1$ & $\mathrm{G} 2$ & $/$ \\
4 & $\mathrm{G} 1$ & $\mathrm{G} 1$ & $\mathrm{G} 2$ & $\mathrm{G} 1$ & $/$ \\
5 & $\mathrm{G} 1$ & $\mathrm{G} 1$ & $\mathrm{G} 2$ & $\mathrm{G} 2$ & $/$ \\
6 & $\mathrm{G} 2$ & $\mathrm{G} 1$ & $\mathrm{G} 2$ & $\mathrm{G} 2$ & $/$ \\
7 & $\mathrm{G} 1$ & $\mathrm{G} 1$ & $\mathrm{G} 2$ & $\mathrm{G} 2$ & $\mathrm{G} 2$ \\
$x_{0}$ & $y_{0}$ & $z_{0}$ & $\alpha_{0}$ & $\beta_{0}$ & $\varphi_{0}$ \\
$\mathrm{G} 1$ & $\mathrm{G} 1$ & $\mathrm{G} 1$ & $\mathrm{G} 1$ & $\mathrm{G} 1$ & $\mathrm{G} 1$ \\
$x_{t}$ & $y_{t}$ & $z_{t}$ & $\alpha_{t}$ & $\beta_{t}$ & $\varphi_{t}$ \\
$\mathrm{G} 1$ & $\mathrm{G} 1$ & $\mathrm{G} 1$ & $\mathrm{G} 1$ & $\mathrm{G} 1$ & $\mathrm{G} 1$ \\
\hline
\end{tabular}

$$
\mathbf{E}^{k}=\mathbf{H}_{r}^{k} \delta \mathbf{q}_{r}
$$

where $\delta \mathbf{q}_{r}$ is the error vector of kinematic parameters in G1, $\mathbf{H}_{r}^{k}$ denotes the corresponding identification Jacobian. It is notable that because of the presence of coupling joint in the 4R 3-DOF wrist, some additional constraints are defined in the controller i.e. $\alpha_{5}=-2 \alpha_{4}, \alpha_{6}=\alpha_{4}$. In other words, the compensation of $\alpha_{4}$ also results changes of $\alpha_{5}$ and $\alpha_{6}$. It means the torsion angle error of joint 4 $\Delta \alpha_{4}$ could result in additional error on torsion angle errors of joint 5 and joint 6 i.e. $\Delta \alpha_{5, \text { add }}=-2 \Delta \alpha_{4}$, $\Delta \alpha_{6, \text { add }}=\Delta \alpha_{4}$. Therefore, the actual differential error of EE results from $\Delta \alpha_{4}$ can be written as

$$
\mathbf{E}_{\Delta \alpha_{4}}^{k}=\left[\mathbf{H}^{k}\left(:, \Delta \alpha_{4}\right)-2 \mathbf{H}^{k}\left(:, \Delta \alpha_{5}\right)+\mathbf{H}^{k}\left(:, \Delta \alpha_{6}\right)\right] \Delta \alpha_{4}
$$

where $\mathbf{H}^{k}\left(:, \Delta \alpha_{4}\right), \mathbf{H}^{k}\left(:, \Delta \alpha_{5}\right), \quad \mathbf{H}^{k}\left(:, \Delta \alpha_{6}\right)$ are respectively columns of the identification Jacobian corresponding to $\Delta \alpha_{4}, \Delta \alpha_{5}$ and $\Delta \alpha_{6}$. Then from Eq. (26), the identification Jacobian considering constrains can derived by adjusting the column corresponding to $\Delta \alpha_{4}$ as

$$
\mathbf{H}_{r}^{k}\left(:, \Delta \alpha_{4}\right)=\mathbf{H}_{r}^{k}\left(:, \Delta \alpha_{4}\right)-2 \mathbf{H}^{k}\left(:, \Delta \alpha_{5}\right)+\mathbf{H}^{k}\left(:, \Delta \alpha_{6}\right)
$$

where $\mathbf{H}_{r}^{k}\left(:, \Delta \alpha_{4}\right)=\mathbf{H}^{k}\left(:, \Delta \alpha_{4}\right)$.

\subsection{Parameter identifiability analysis}

To avoid potential singularity problem in solving Eq. (17), parameter identifiability analysis is conducted in this section, and the redundant parameters would be removed before practical parameter identification. Here, the QR decomposition technique is utilized to solve this problem. According to QR decomposition, the identification Jacobian $\mathbf{H}$ can be rewritten as

$$
\mathbf{H}_{m \times c}=\mathbf{Q}_{m \times m}\left[\begin{array}{c}
\mathbf{R}_{c \times c} \\
\mathbf{0}_{(m-c) \times c}
\end{array}\right]
$$

where $\mathbf{Q}_{m \times m}$ is a $m \times m$ orthogonal matrix, $\mathbf{R}_{c \times c}$ is a $c \times c$ upper triangular matrix, and $\mathbf{0}_{(m-c) \times c}$ is a $(m-\mathrm{c}) \times \mathrm{c}$ null matrix. Then, Eq. (17) can be rewritten as

$$
\mathbf{E}_{m \times 1}=\mathbf{Q}_{m \times m}\left[\begin{array}{c}
\mathbf{R}_{c \times c} \\
\mathbf{0}_{(m-c) \times c}
\end{array}\right] \delta \mathbf{q}_{c \times 1}
$$

where $m=6 N$ is the number of scalar equations, $c$ is the number of unknown parameters (for original MDH model $c=42$, for reduced model $c=28$ ). From Eq. (29), the unknown parameters whose corresponding elements on the diagonal of $\mathbf{R}_{c \times c}$ are zero can be regarded as redundant. It is notable that the redundant parameters whose corresponding columns of $\mathbf{R}_{c \times c}$ are all zeroes have no effect on differential error of EE, while other redundant parameters are linear correlation with one or more identifiable parameters. And for several linearly dependent parameters, the first $k$ independent 
parameters in the vector will be given as the identifiable ones. Therefore, it is worthwhile to rearrange the error vector of kinematic parameters according to complexity of their compensation.

After the redundant parameters are obtained, they are removed to ensure identification Jacobian invertible. If $r$ is the rank of $\mathbf{H}$, there are $c-r$ redundant parameters. For simplicity, the error vector of kinematic parameters after removing the redundant parameters is noted as $\delta \mathbf{q}_{a}$, and the identification Jacobian after removing corresponding columns is noted as $\mathbf{H}_{a}$. Then the final identification model is expressed as

$$
\mathbf{E}^{k}=\mathbf{H}_{a}^{k} \delta \mathbf{q}_{a}
$$

Following the idea above, for original MDH model, $r=34$. There are 8 redundant parameters in $\delta \mathbf{q}$ : $\delta d_{3}, \delta z_{0}, \delta \varphi_{0}, \delta x_{t}, \delta z_{t}, \delta \alpha_{t}, \delta \beta_{t}, \delta \varphi_{t}$. The final identifiable parameter vector is

$$
\begin{aligned}
\delta \mathbf{q}_{a}= & {\left[\delta \theta_{1}, \delta d_{1}, \delta a_{1}, \delta \alpha_{1}, \delta \theta_{2}, \delta d_{2}, \delta a_{2}, \delta \alpha_{2}, \delta \beta_{2}, \delta \theta_{3}, \delta a_{3}, \delta \alpha_{3}, \delta \theta_{4}, \delta d_{4},\right.} \\
& \delta a_{4}, \delta \alpha_{4}, \delta \theta_{5}, \delta d_{5}, \delta a_{5}, \delta \alpha_{5}, \delta \theta_{6}, \delta d_{6}, \delta a_{6}, \delta \alpha_{6}, \delta \theta_{7}, \delta d_{7}, \delta a_{7}, \delta \alpha_{7}, \\
& \left.\delta \beta_{7}, \delta x_{0}, \delta y_{0}, \delta \alpha_{0}, \delta \beta_{0}, \delta y_{t}\right]^{T}
\end{aligned}
$$

While for reduced model, $r=24$. There are 4 redundant parameters in $\delta \mathbf{q}_{r}: \delta z_{0}, \delta \varphi_{0}, \delta z_{t}, \delta \varphi_{t}$. The final identifiable parameter vector is

$$
\begin{gathered}
\delta \mathbf{q}_{r, a}=\left[\delta \theta_{1}, \delta \theta_{2}, \delta \theta_{3}, \delta \theta_{4}, \delta \theta_{5}, \delta \theta_{7}, \delta d_{1}, \delta d_{2}, \delta d_{4}, \delta d_{5}, \delta d_{6}, \delta d_{7}, \delta a_{1}, \delta a_{2},\right. \\
\left.\delta a_{3}, \delta \alpha_{4}, \delta x_{0}, \delta y_{0}, \delta \alpha_{0}, \delta \beta_{0}, \delta x_{t}, \delta y_{t}, \delta \alpha_{t}, \delta \beta_{t}\right]^{T}
\end{gathered}
$$

\section{Experiments and discussion}

To verify effectiveness of the proposed method, the kinematic calibration experiment of a 7R 6-DOF painting robot as shown in Figure 1 has been conducted. In the experiment, the measurement device is a LEICA AT901-LR laser tracker with the maximum observed error of $5 \mu \mathrm{m} / \mathrm{m}$. It is notable that the proposed method needs measurements of full pose of EE (three translations and three rotations) while the laser tracker with a reflector generally offers the position (three translations) i.e. the center of a sphere reflector. Therefore, a specific end-effector tool has been designed in this paper, as shown in Figure 2. The tool is bolted to the end flange of the robot, and it has four reflector holders, the centers of which make a rectangle. Then in order to get full pose of EE, at least three reflectors should be measured at each robot configuration. For simplicity, a tool coordinate system is defined as shown in Figure 2 (b). The original point is located on the center point of the rectangle i.e. the center of line $\mathrm{P}_{2} \mathrm{P}_{3}$, and can be expressed as

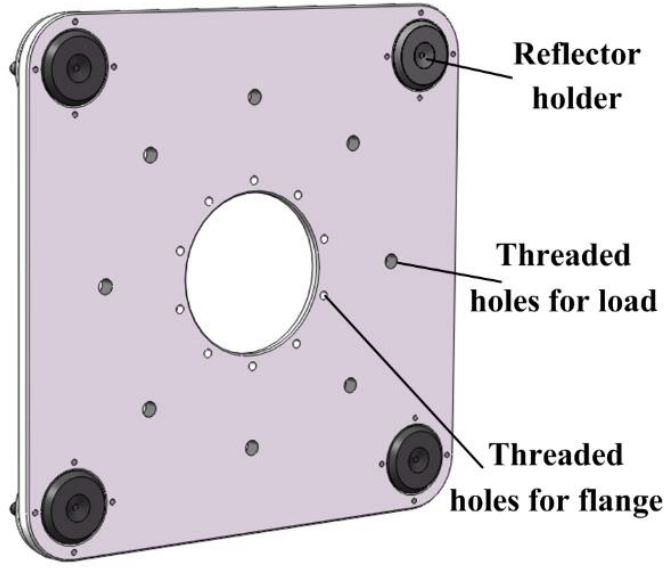

(a)

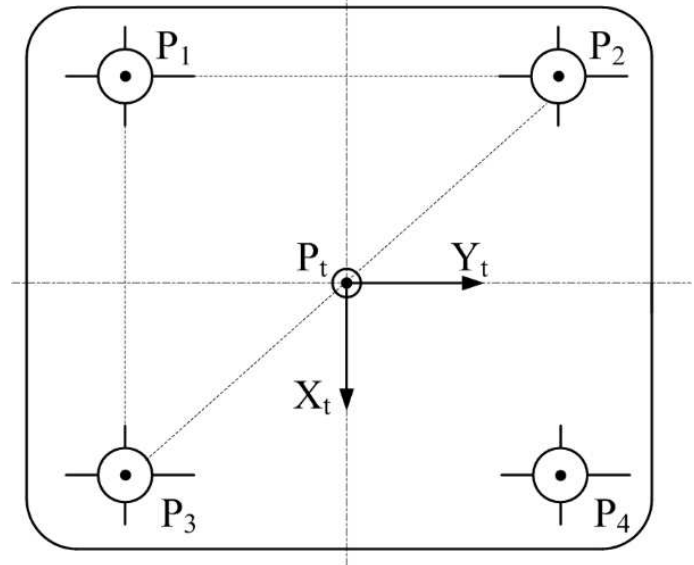

(b)

Figure 2 End-effector tool for kinematic calibration (a) 3D (b) Tool coordinate system

$$
\mathbf{P}_{t}=\left(\mathbf{P}_{2}+\mathbf{P}_{3}\right) / 2
$$

where $\mathbf{P}_{t}$ is the position vector of tool frame. The $\mathrm{Z}$ axis is defined as the normal vector of plane $\mathrm{P}_{1} \mathrm{P}_{2} \mathrm{P}_{3}$, $\mathrm{Y}$ axis is aligned on line $\mathrm{P}_{1} \mathrm{P}_{2}$, while $\mathrm{X}$ axis is derived according to the right-hand rule. The measured tool transformation matrix can be written as

$$
{ }_{\text {tool }}^{\text {base }} \mathbf{T}^{r}=\left[\begin{array}{cc}
\mathbf{R}_{t} & \mathbf{P}_{t} \\
0 & 1
\end{array}\right]
$$


where $\mathbf{R}_{t}=\left[\mathbf{r}_{x}, \mathbf{r}_{y}, \mathbf{r}_{z}\right]$ is the orientation matrix of tool frame. And the elements are defined as

$$
\begin{aligned}
& \mathbf{r}_{y}=\left(\mathbf{P}_{2}-\mathbf{P}_{1}\right) /\left\|\mathbf{P}_{2}-\mathbf{P}_{1}\right\| \\
& \mathbf{r}_{z}=\left[\left(\mathbf{P}_{3}-\mathbf{P}_{1}\right) /\left\|\mathbf{P}_{3}-\mathbf{P}_{1}\right\|\right] \times \mathbf{r}_{y} \\
& \mathbf{r}_{x}=\mathbf{r}_{y} \times \mathbf{r}_{z}
\end{aligned}
$$

The experimental set-up for kinematic calibration of the 7R 6-DOF painting robot is illustrated in Figure 3. In order to clearly describe the experiment procedure, the steps are expressed as follows:

1) Select $N$ robot configurations $\boldsymbol{\theta}=\left[\theta_{1}, \theta_{2}, \theta_{3}, \theta_{4}, \theta_{5}, \theta_{7}\right]$ for pose measurement, they should be randomly distributed in the robot' usable workspace and ensure at least three reflectors are visible by the laser tracker.

2) The robot is commanded to undergo the measurement configurations successively, the position data of three reflectors are captured by the laser tracker. Then differential error vectors $\mathbf{E}^{k}$ of EE are derived. The measurement at each configuration is repeated three times and the mean value is retained.

3) From Eqs. (20), (21) and (24), the normal base transformation matrix $\mathbf{T}_{\text {base }}$ is estimated.

4) From Eq. (18), the error vector of kinematic parameters $\delta \mathbf{q}$ can be obtained, it is notable that the original identification matrix $\mathbf{H}_{a}$ and reduced one $\mathbf{H}_{r, a}$ are respectively utilized for comparison.

5) According to the identified kinematic parameters, error compensation is conducted and analyzed. It is worthwhile to point out that the errors of reduced model can be compensated by directly modification of parameters in the controller, while that of the original model should be compensated by adjusting the controller input i.e. target configurations. For error compensation of the original model, the target configurations can be adjusted by

$$
{ }_{\text {tool }}^{\text {base }} \mathbf{T}{ }_{a}={ }_{\text {tool }}^{\text {base }} \mathbf{T}\left(\mathbf{I}+\boldsymbol{\Delta}_{\text {original }}\right)
$$

where ${ }_{\text {tool }}^{\text {base }} \mathbf{T},{ }_{\text {tool }}^{\text {base }} \mathbf{T}$ are respectively the original and adjusted target configurations, $\boldsymbol{\Delta}_{\text {original }}$ is the differential operator of EE calculated by the original model.

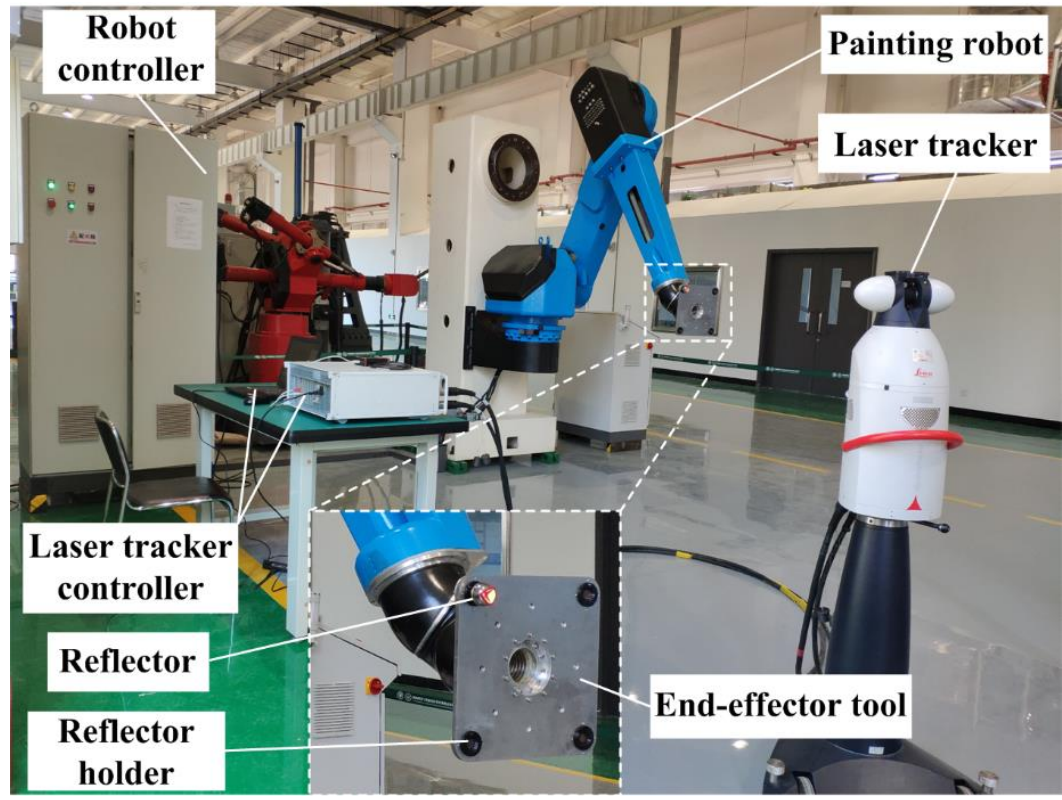

Figure 3 Experiment set-up for kinematic calibration of the 7R 6-DOF painting robot

According to analysis in Section 3.2, there are 34 and 24 kinematic parameters respectively for the original model and the reduced model need to be identified. Then at least 6 measurement configurations are needed i.e. $N \geq 6$. In order to reduce effects of measurement errors on parameter identification accuracy, 50 different measurement configurations are selected in the practical experiment.

From Eqs. (20), (21) and (24), the normal base transformation matrix $\mathbf{T}_{\text {base }}$ is firstly estimated by using the measured data. For comparison, the base transformation matrix is also identification by additional measuring the axes 1 and 2 [30], as shown in Figure 4. Additional two reflectors are respectively fixed on the waist and upperarm of the robot, by respectively rotating joint 1 and joint2 (with the others being locked) and capturing positions of the reflectors by the laser tracker, the axes along joint 1 and joint 2 can be derived. Then, the base coordinate system is defined as shown in Figure 4 (b). For simplicity, the 
obtained base kinematic parameters by the two methods (the proposed method and additional measurement method) are summarized in Table 3.

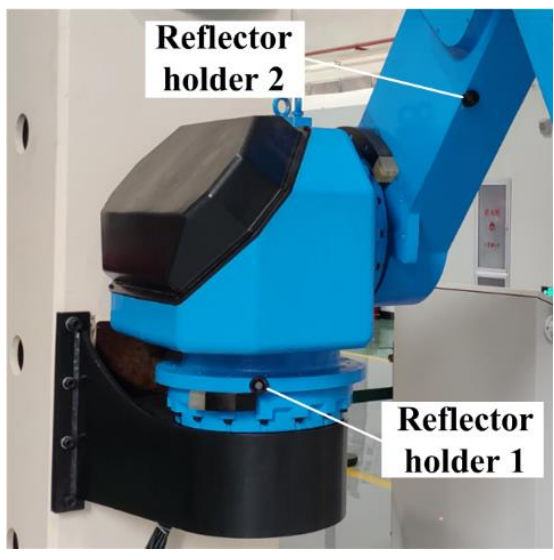

(a)

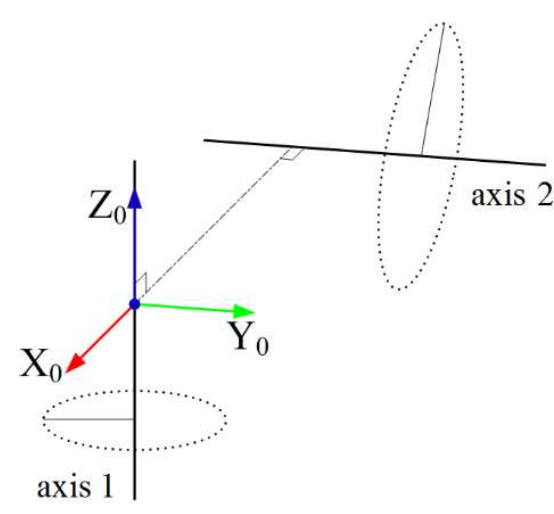

(b)

Figure 4 Measurement of the base frame (a) experiment set-up (b) defining the base coordinate system

Table 3 Base kinematic parameters of the robot

\begin{tabular}{ccccccc}
\hline$/$ & $x_{0}(\mathrm{~mm})$ & $y_{0}(\mathrm{~mm})$ & $z_{0}(\mathrm{~mm})$ & $\alpha_{0}(\mathrm{deg})$ & $\beta_{0}(\mathrm{deg})$ & $\varphi_{0}(\mathrm{deg})$ \\
\hline $\begin{array}{c}\text { Proposed method } \\
\text { Additional measurement } \\
\text { method }\end{array}$ & -462.9100 & 4097.4547 & -197.7500 & 0.6486 & 0.3299 & -59.4643 \\
& -463.1357 & 4095.966 & -195.6047 & 0.4100 & 0.3372 & -59.6511 \\
\hline
\end{tabular}

From Table 3, errors between the base kinematic parameters by the two methods are really small with the maximum translational and orientational errors are respectively $2.15 \mathrm{~mm}$ and $0.24^{\circ}$. These errors can be identified and compensated in the following steps of the calibration. In other words, the proposed method for base frame estimation is feasible, and it is more suitable for industrial application because no additional measurements are needed, which is an advantage of the proposed calibration method.

After estimation of the base frame, from Eq. (18), the kinematic parameter errors can be identified. For comparison, the original MDH model and reduced model are both utilized, and the results are shown in Table 4.

Table 4 Results of kinematic parameter identification of the robot

\begin{tabular}{cccc}
\hline$/$ & $\begin{array}{c}\text { Normal value } \\
\mathbf{q}(\mathrm{mm}, \mathrm{deg})\end{array}$ & $\begin{array}{c}\text { Original model } \\
\mathbf{q}_{a}(\mathrm{~mm}, \mathrm{deg})\end{array}$ & $\begin{array}{c}\text { Reduced model } \\
\mathbf{q}_{r, a}(\mathrm{~mm}, \mathrm{deg})\end{array}$ \\
\hline$\theta_{1}$ & 0 & 0.3573 & 0.1626 \\
$d_{1}$ & 0 & 4.2324 & 4.6001 \\
$a_{1}$ & 0 & 0.1888 & 0.9890 \\
$\alpha_{1}$ & -90 & -90.0304 & -90 \\
$\theta_{2}$ & 0 & -0.2339 & -0.2440 \\
$d_{2}$ & 0 & -4.9113 & -2.8339 \\
$a_{2}$ & 1300 & 1302.1207 & 1302.0667 \\
$\alpha_{2}$ & 0 & -0.0150 & 0 \\
$\beta_{2}$ & 0 & 0.1747 & 0 \\
$\theta_{3}$ & 0 & -0.0137 & -0.0358 \\
$d_{3}$ & 0 & 0 & 0 \\
$a_{3}$ & 0 & -4.1197 & -5.0991 \\
$\alpha_{3}$ & -90 & -90.1729 & -90 \\
$\theta_{4}$ & 0 & -0.2249 & 0.1229 \\
$d_{4}$ & 1474 & 1471.5227 & 1471.9281
\end{tabular}




\begin{tabular}{lccc}
$a_{4}$ & 0 & 2.3213 & 0 \\
$\alpha_{4}$ & -35 & -34.9859 & -34.8464 \\
$\theta_{5}$ & 0 & 2.1370 & 1.9169 \\
$d_{5}$ & 95 & 99.7417 & 98.1055 \\
$a_{5}$ & 0 & -1.6359 & 0 \\
$\alpha_{5}$ & 70 & 69.8252 & 70 \\
$\theta_{6}$ & 0 & -1.6310 & 0 \\
$d_{6}$ & 95 & 102.3560 & 100.7109 \\
$a_{6}$ & 0 & -1.0774 & 0 \\
$\alpha_{6}$ & -35 & -34.5065 & -35 \\
$\theta_{7}$ & 0 & -0.0775 & 0.0958 \\
$d_{7}$ & 85 & 81.5858 & 82.8581 \\
$a_{7}$ & 0 & -1.0380 & 0 \\
$\alpha_{7}$ & 0 & -0.3838 & 0 \\
$\beta_{7}$ & 0 & 2.1484 & 0 \\
$x_{0}$ & -462.9100 & -464.5854 & -464.8290 \\
$y_{0}$ & 4097.4547 & 4101.0744 & 4101.5041 \\
$z_{0}$ & -197.7500 & -197.7500 & -197.7500 \\
$\alpha_{0}$ & 0.6486 & 0.9123 & 0.9307 \\
$\beta_{0}$ & 0.3299 & 0.4198 & 0.4376 \\
$\varphi_{0}$ & -59.4643 & -59.4643 & -59.4643 \\
$x_{t}$ & 0 & 0 & -1.1824 \\
$y_{t}$ & 25.6200 & 24.1504 & 24.8826 \\
$z_{t}$ & 0 & 0 & 0 \\
$\alpha_{t}$ & 0 & 0 & 2.0693 \\
$\beta_{t}$ & 0 & 0 & 0 \\
$\varphi_{t}$ & 0 & 0 & \\
\hline & & & 0.4935 \\
\hline & 0 & 0 & 0 \\
\hline
\end{tabular}

Finally, according to the identified kinematic parameters, error compensation is conducted and analyzed. For the reduced model, errors can be compensated by directly modifying parameters in the controller, while the errors of the original model should be compensated by adjusting the target configurations i.e. Eq. (38). The pose errors of the 50 measurement configurations before and after calibration are shown in Figure 5. For simplicity, the position and orientation errors of the robot before and after calibration are also respectively summarized in Table 5 and Table 6.

From Figure 5, Table 5 and Table 6, both of the calibration methods based on the original MDH model and reduced model can reduce the pose errors of EE of the 7R 6-DOF painting robot. After kinematic calibration based on the original MDH model and reduced model, the maximum position errors are reduced from $7.2824 \mathrm{~mm}$ respectively to $1.8561 \mathrm{~mm}$ and $1.9436 \mathrm{~mm}$, the average position errors are reduced from $4.6786 \mathrm{~mm}$ respectively to $0.7693 \mathrm{~mm}$ and $0.8313 \mathrm{~mm}$, the maximum orientation errors are reduced from $1.6496^{\circ}$ respectively to $0.3925^{\circ}$ and $0.4001^{\circ}$, the average orientation errors are reduced from $1.0707^{\circ}$ respectively to $0.1458^{\circ}$ and $0.1493^{\circ}$. Generally speaking, the method based on the original model could get a better result than that based on the reduced model. However, the difference is not so obvious and the compensation of the later is easier. In other words, the proposed kinematic calibration method based on the reduced MDH model is more suitable for practical industrial application, which also validates the rationality of model reduction proposed in this paper. 


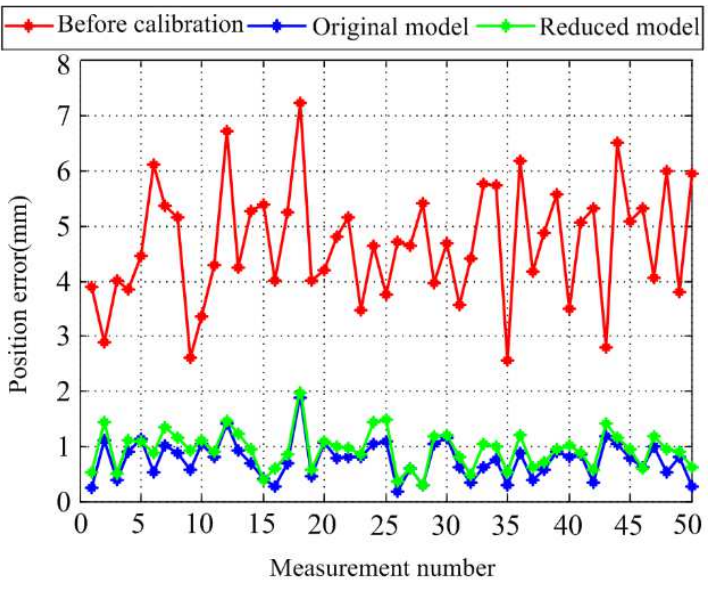

(a)

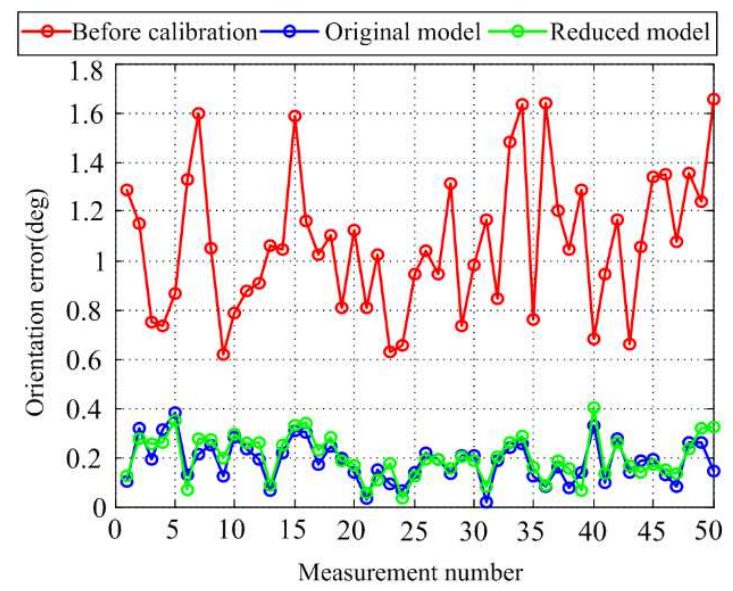

(b)

Figure 5 Pose errors of the robot before and after calibration (a) position error (b) orientation error

Table 5 The position errors of the robot before and after calibration

\begin{tabular}{ccc}
\hline$/$ & Maximum $(\mathrm{mm})$ & Average $(\mathrm{mm})$ \\
\hline Before calibration & 7.2824 & 4.6786 \\
Original model & 1.8561 & 0.7693 \\
Reduced model & 1.9436 & 0.8313 \\
\hline
\end{tabular}

Table 6 The orientation errors of the robot before and after calibration

\begin{tabular}{ccc}
\hline$/$ & Maximum $(\mathrm{deg})$ & Average $(\mathrm{deg})$ \\
\hline Before calibration & 1.6496 & 1.0707 \\
Original model & 0.3925 & 0.1458 \\
Reduced model & 0.4001 & 0.1493 \\
\hline
\end{tabular}

\section{Conclusions}

Kinematic calibration problem of a 7R 6-DOF robot with non-spherical wrist is studied in this paper. The main contributions are the parameters identifiability analysis and model reduction in the identification procedure. Firstly, the kinematic error model is constructed based on the modified DH (MDH) model. Secondly, in order to derive a minimal model which is more suitable for practical industrial application, model reduction is implemented considering the compensability and constraints of the parameters defined in the controller, and QR decomposition is utilized to deal with the parameters identifiability analysis. Finally, to simplify the process of calibration with laser tracker, a two-step procedure for error parameter estimation is proposed: (1) estimation of the robot base location with respect to the measurement coordinate system; (2) fine estimation of the identifiable kinematic parameters according to the measurement data.

In order to validate the effectiveness of the proposed method, the calibration experiment on a 7R 6DOF industrial painting robot is implemented. The results show that the average position errors and orientation errors are reduced from $4.6786 \mathrm{~mm}$ and $1.0707^{\circ}$ respectively to $0.8313 \mathrm{~mm}$ and $0.1493^{\circ}$, which shows that the proposed method can effectively improve the accuracy of the robot. Additionally, because parameter errors can be compensated by directly modification of the model parameters in the controller, the proposed kinematic calibration method based on the reduced $\mathrm{MDH}$ model is more suitable for practical industrial application, which is the main advantage of the proposed method. To further improve accuracy of the robot, our further work will be carried out to investigate the non-kinematic calibration method of the 7R 6-DOF robot.

\section{Declarations}

Availability of data and materials

Not applicable.

\section{Acknowledgements}

Not applicable. 


\section{Authors' contributions}

WX was in charge of the whole analyses. ZD and ZC wrote the manuscript. GZ, WM, MX and ML assisted with sampling and laboratory analyses. All authors read and approved the final manuscript.

\section{Authors' Information}

Xuhao Wang, born in 1989, is currently a lecturer at Civil Aviation University of China, China. He received his $\mathrm{PhD}$ degree from Tianjin University, China, in 2020. His research interests include industrial painting robot, robotics.

Dawei Zhang, born in 1962, is currently a professor at the School of Mechanical Engineering, Tianjin University, China. He received his $\mathrm{PhD}$ degree from Tianjin University, China, in 1995. His research interests include dynamic design of machine tools, micro-nano manufacturing, design and application of industrial painting robot.

Chen Zhao, born in 1963, is currently an associate professor at Tianjin University, China. He received his $\mathrm{PhD}$ degree from Franche-Comte University, France, in 1997. His research interests include design and control of industrial painting robot, medical robot system.

\section{Funding}

This work is financially supported by Tianjin Science and Technology Committee (Grant No. 15ZXZNGX00200), Fundamental Research Funds for the Central Universities (Grant No. 3122020030), and Joint Funds of the National Natural Science Foundation of China \& Civil Aviation Administration of China (Grant No. U1833106).

\section{Competing interests}

The authors declare that they have no competing interests.

\section{Author Details}

${ }^{1}$ Aeronautical Engineering College, Civil Aviation University of China, Tianjin, 300300, China. ${ }^{2}$ Key Laboratory of Mechanism Theory and Equipment Design of Ministry of Education, School of Mechanical Engineering, Tianjin University, Tianjin, 300350, China. ${ }^{3}$ Automotive Engineering Corporation, Tianjin, 300113, China.

\section{Appendix A. The transform matrix between $\mathbf{e}_{i}$ and $\delta \mathbf{q}_{i}$}

$$
\begin{aligned}
\mathbf{M}_{i} & =\left[\begin{array}{llll}
0 & 0 & 1 & 0 \\
a_{i} c \alpha_{i} & s \alpha_{i} & 0 & 0 \\
-a_{i} s \alpha_{i} & c \alpha_{i} & 0 & 0 \\
0 & 0 & 0 & 1 \\
s \alpha_{i} & 0 & 0 & 0 \\
c \alpha_{i} & 0 & 0 & 0
\end{array}\right], i=1,3,4,5,6 \\
\mathbf{M}_{i} & =\left[\begin{array}{lllll}
a_{i} s \alpha_{i} s \beta_{i} & -c \alpha_{i} s \beta_{i} & c \beta_{i} & 0 & 0 \\
a_{i} c \alpha_{i} & s \alpha_{i} & 0 & 0 & 0 \\
-a_{i} s \alpha_{i} c \beta_{i} & c \alpha_{i} c \beta_{i} & s \beta_{i} & 0 & 0 \\
-c \alpha_{i} s \beta_{i} & 0 & 0 & c \beta_{i} & 0 \\
s \alpha_{i} & 0 & 0 & 0 & 1 \\
c \alpha_{i} c \beta_{i} & 0 & 0 & s \beta_{i} & 0
\end{array}\right], i=2,7 \\
\mathbf{M}_{i} & =\left[\begin{array}{lllllll}
c \beta_{i} c \varphi_{i} & c \alpha_{i} s \varphi_{i}+c \varphi_{i} s \alpha_{i} s \beta_{i} & s \alpha_{i} s \varphi_{i}-c \varphi_{i} c \alpha_{i} s \beta_{i} & 0 & 0 & 0 \\
-c \beta_{i} s \varphi_{i} & c \alpha_{i} c \varphi_{i}-s \varphi_{i} s \alpha_{i} s \beta_{i} & s \alpha_{i} c \varphi_{i}+s \varphi_{i} c \alpha_{i} s \beta_{i} & 0 & 0 & 0 \\
s \beta_{i} & -c \beta_{i} s \alpha_{i} & c \beta_{i} c \alpha_{i} & 0 & 0 & 0 \\
0 & 0 & 0 & c \beta_{i} c \varphi_{i} & s \varphi_{i} & 0 \\
0 & 0 & 0 & -c \beta_{i} s \varphi_{i} & c \varphi_{i} & 0 \\
0 & 0 & 0 & 1
\end{array}\right], i=0, t
\end{aligned}
$$




\section{References}

[1] N Albert, A B Ilian. Absolute calibration of an ABB IRB 1600 robot using a laser tracker. Robotics and Computer Integrated Manufacturing, 2013, 29: 236-245.

[2] T Huang, D Zhao, F W Yin, et al. Kinematic calibration of a 6-DOF hybrid robot by considering multicollinearity in the identification Jacobian. Mechanism and Machine Theory, 2019, 131: 371384.

[3] A Y Elatta, L P Gen, F L Zhi, et al. An overview of robot calibration. Information Technology Journal, 2004, 3(1): 74-78.

[4] G Chen, $\mathrm{T} \mathrm{Li}, \mathrm{M}$ Chu, et al. Review on kinematics calibration technology of serial robots. International Journal of Precision Engineering and Manufacturing. 2014, 15: 1759-1774.

[5] T Sun, Y P Zhai, Y M Song, et al. Kinematic calibration of a 3-DoF rotational parallel manipulator using laser tracker. Robotics and Computer Integrated Manufacturing, 2016, 41: 78-91.

[6] K Schröer, S L Albright, M Grethlein. Complete, minimal and model-continuous kinematic models for robot calibration. Robotics and Computer Integrated Manufacturing, 1997, 13(1): 73-85.

[7] J Denavit, R S Hartenberg. A kinematic notation for lower-pair mechanisms based on matrices. Journal of Applied Mechanics, 1955, 22: 215-221.

[8] T Messay, R Ordóñez, E. Marcil. Computationally efficient and robust kinematic calibration methodologies and their application to industrial robots. Robot. Robotics and Computer Integrated Manufacturing, 2016, 37: 33-48.

[9] S Lee, Q Zeng, K F Ehmann. Error modeling for sensitivity analysis and calibration of the tripyramid parallel robot. International Journal of Advanced Manufacturing Technology, 2019, 93: 1319-1332.

[10] S A Hayati. Robot arm geometric link parameter estimation. Proceedings of 22th IEEE conference on decision control, (1983), pp. 1477-83.

[11] H W Stone, A C Sanderson. Statistical performance evaluation of the S-model arm signature identification technique. Proceedings of the 1988 IEEE International Conference on Robotics and Automation, (1988), pp. 939-946.

[12] B W Mooring, G R Tang. Improved method for identifying the kinematic parameters in a six axis robot. Proceedings of the International Computers in Engineering Conference and Exhibit, (1984), pp: 79-84.

[13] H Zhuang, Z S Roth, F Hamano. A complete and parametrically continuous kinematic model for robot manipulators. IEEE Transactions on Robotics and Automation, 1992, 8: 451-463.

[14] R B He, Y J Zhao, S N Yang, et al. Kinematic-parameter identification for serial-robot calibration based on POE formula. IEEE Transactions on Robotics, 2010, 26(3):411-423.

[15] X D Yang, L Wu, J Q Li, et al. A minimal kinematic model for serial robot calibration using POE formula. Robotics and Computer Integrated Manufacturing, 2014, 30: 326-334

[16] W Khalil, E Dombre. Modeling, identification and control of robots. Butter-worth-Heinemann, Oxford, 2004.

[17] G B Gao, G Q Sun, J Na, et al. Structural parameter identification for 6 DOF industrial robots. Mechanical Systems and Signal Processing, 2018, 113: 145-155.

[18] A Joubair, I A Bonev. Kinematic calibration of a six-axis serial robot using distance and sphere constraints. International Journal of Advanced Manufacturing Technology, 2015, 77:515-523.

[19] R Wang, A Wu, X Chen, et al. A point and distance constraint based 6R robot calibration method through machine vision. Robotics and Computer Integrated Manufacturing, 2020, 65: 1-7.

[20] Y Wu, A Klimchik, S Caro, et al. Geometric calibration of industrial robots using enhanced partial pose measurements and design of experiments. Robotics and Computer Integrated Manufacturing, 2015, 35: 151-168.

[21] W Wang, L Wang, C Yun. Design of a two-step calibration method of kinematic parameters for serial robots. Chinese Journal of Mechanical Engineering, 2017, 30: 438-448.

[22] N Albert, A B Ilian. Absolute robot calibration with a single telescoping ballbar. Precision Engineering, 2014, 38: 472-480.

[23] M Russo, X Dong. A calibration procedure for reconfigurable Gough-Stewart manipulators. Mechanism and Machine Theory, 2020, 152: 1-14.

[24] A Filion, A Joubair, A S Tahan, et al. Robot calibration using a portable photogrammetry system. Robotics and Computer Integrated Manufacturing, 2018, 49: 77-87.

[25] S Kolyubin, A Shiriaev, A Jubien. Consistent kinematics and dynamics calibration of lightweight redundant industrial manipulators. International Journal of Advanced Manufacturing Technology, 2019, 101: 243-259.

[26] Y Wu, A Klimchik, A Pashkevich, et al. Efficiency improvement of measurement pose selection techniques in robot calibration. IFAC Proceedings Volumes, 2013, 46(9): 802-807. 
[27] Q X Jia, S W Wang, G Chen, et al. A novel optimal design of measurement configurations in robot calibration. Mathematical Problems in Engineering, 2018, 2018: 1-17.

[28] X H Wang, D W Zhang, C Zhao. The inverse kinematics of a 7R 6-degree-of-freedom robot with non-spherical wrist. Advances in Mechanical Engineering, 2017, 9(8): 1-11.

[29] T Huang, H T Liu, D G Chetwynd. Generalized Jacobian analysis of lower mobility manipulators. Mechanism and Machine Theory, 2011, 46(6): 831-844.

[30] J B Zhang, X B Wang, K Wen, et al. A simple and rapid calibration methodology for industrial robot based on geometric constraint and two-step error. Industrial Robot: An International Journal, 2018, 45(6): 715-721. 
Figures

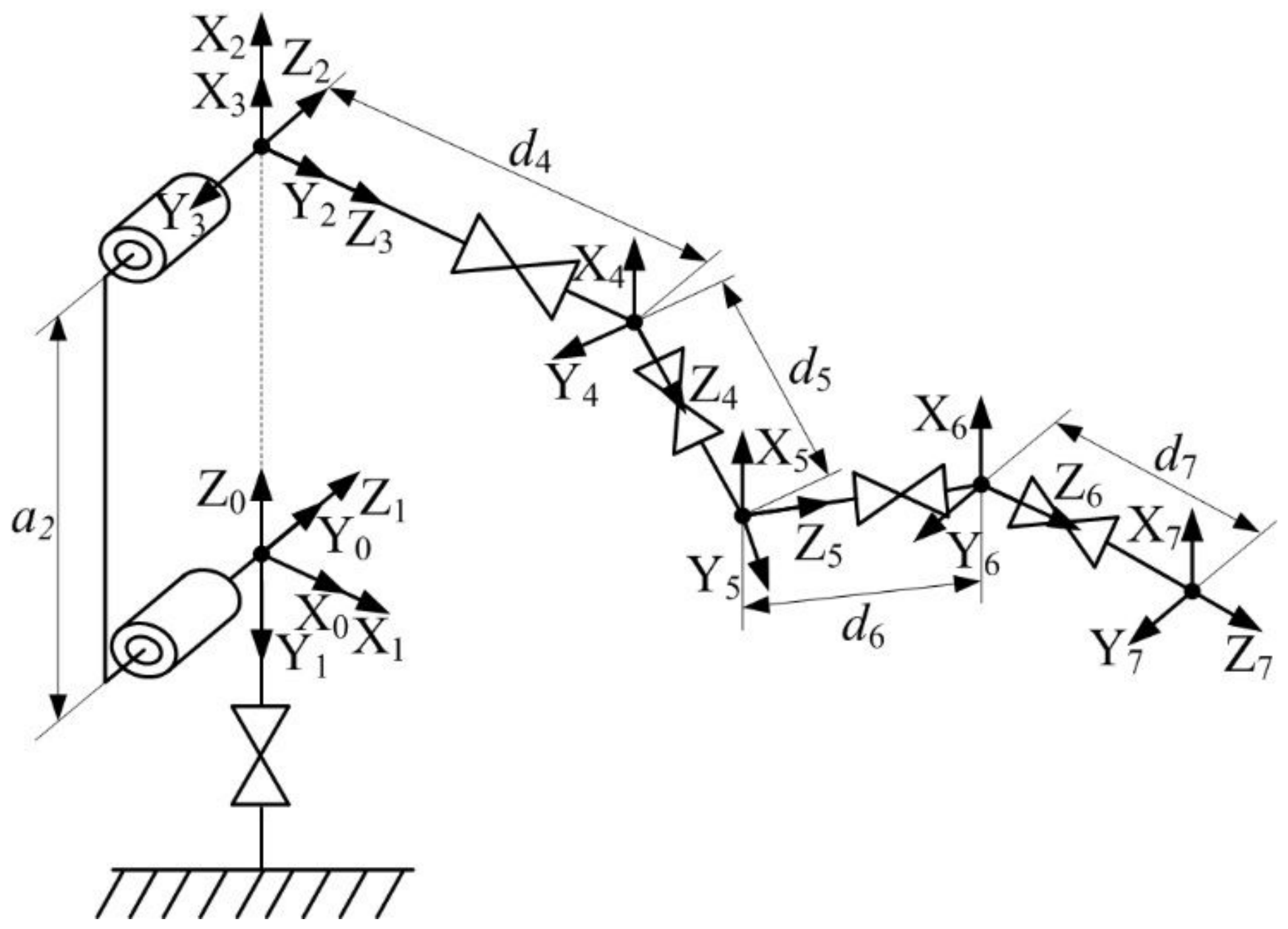

Figure 1

The 7R 6-DOF painting robot with non-spherical wrist (a) Virtual prototype (b) Schematic diagram and coordinate systems 


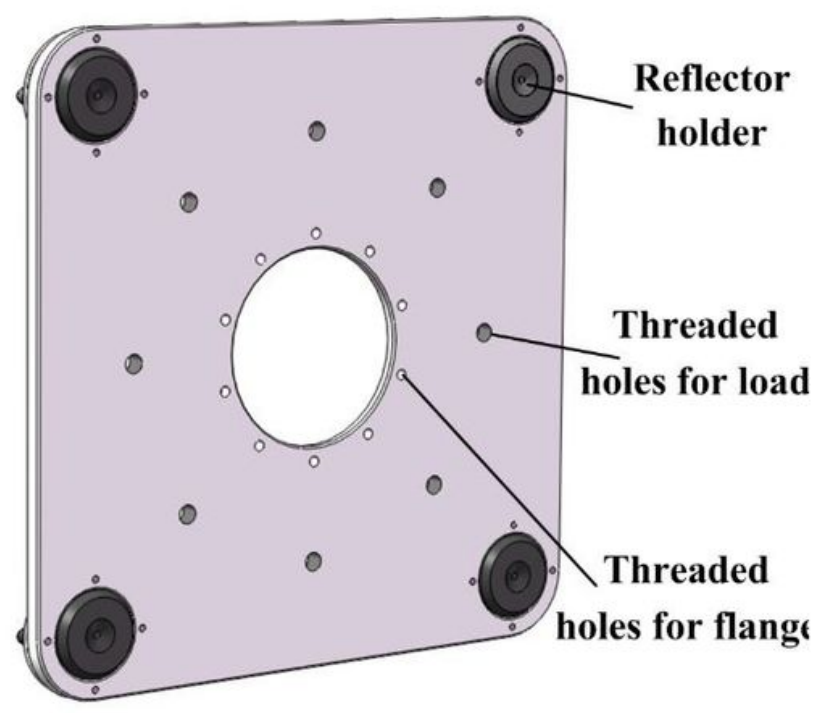

(a)

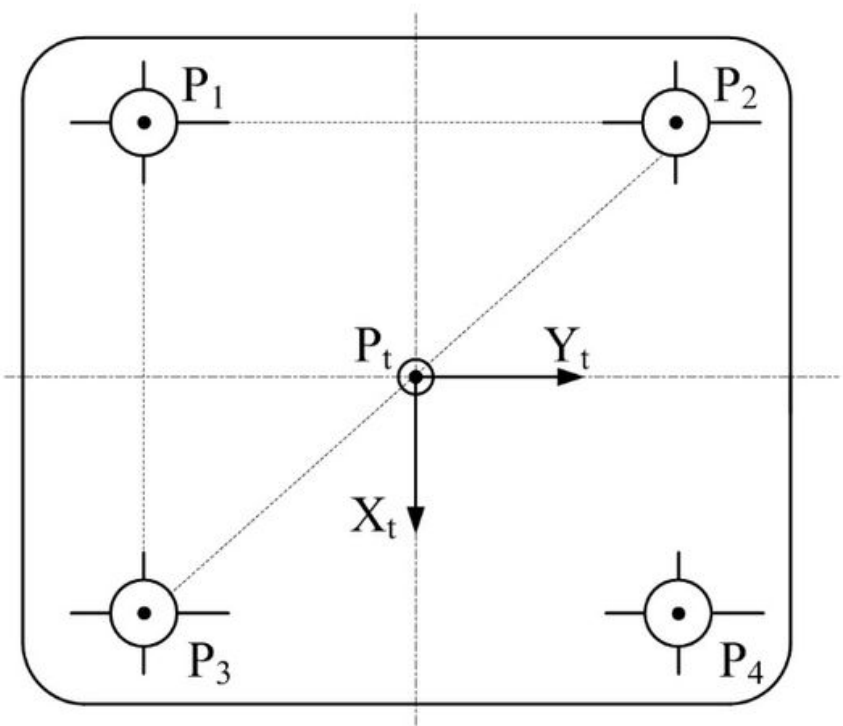

(b)

Figure 2

End-effector tool for kinematic calibration (a) 3D (b) Tool coordinate system 


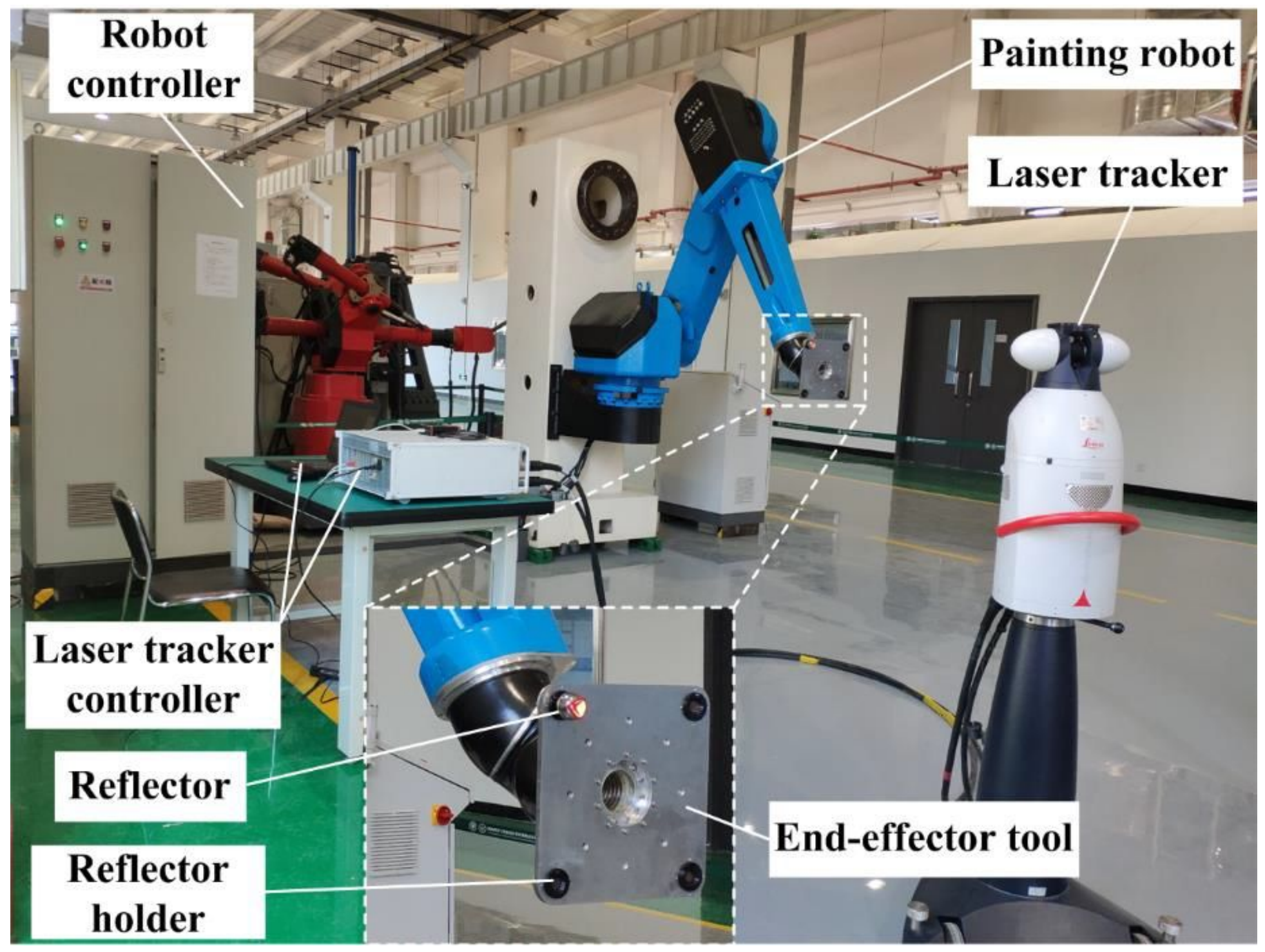

Figure 3

Experiment set-up for kinematic calibration of the 7R 6-DOF painting robot 


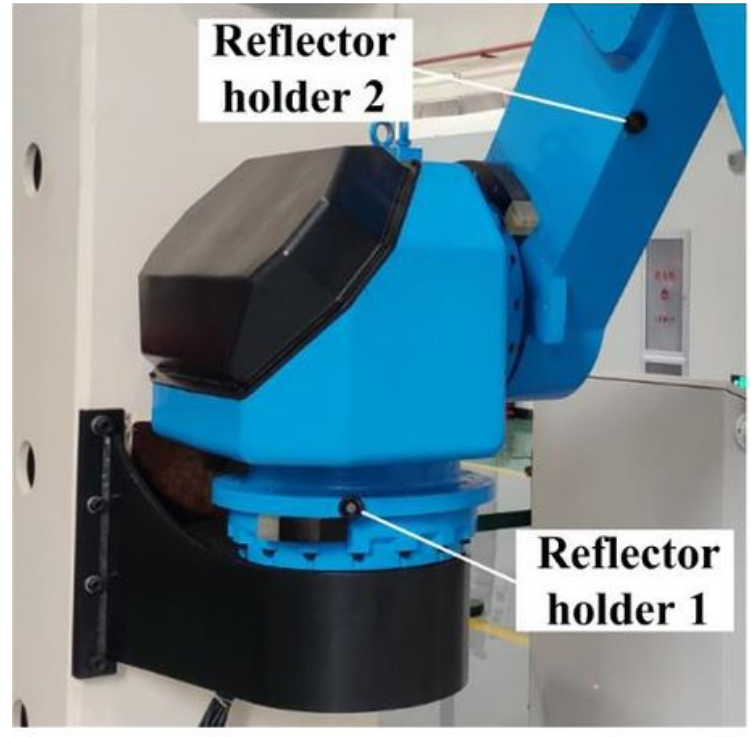

(a)

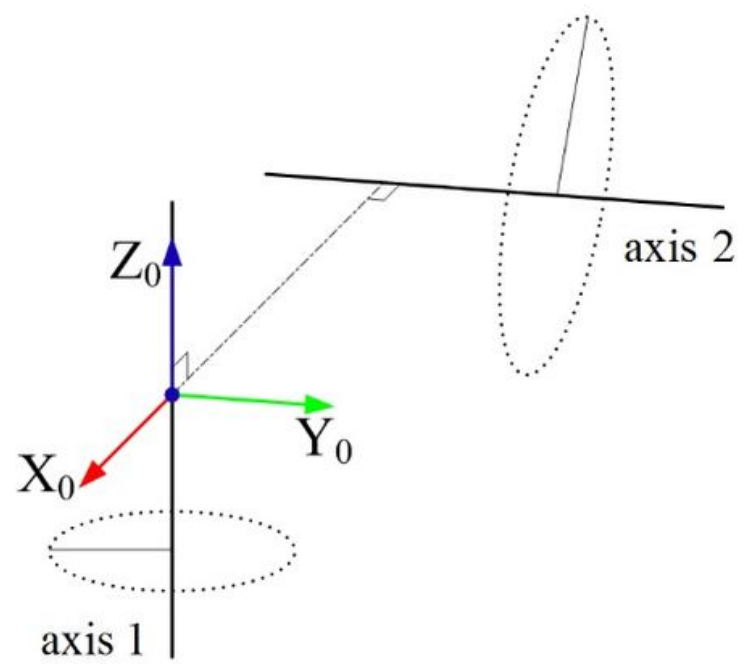

(b)

Figure 4

Measurement of the base frame (a) experiment set-up (b) defining the base coordinate system

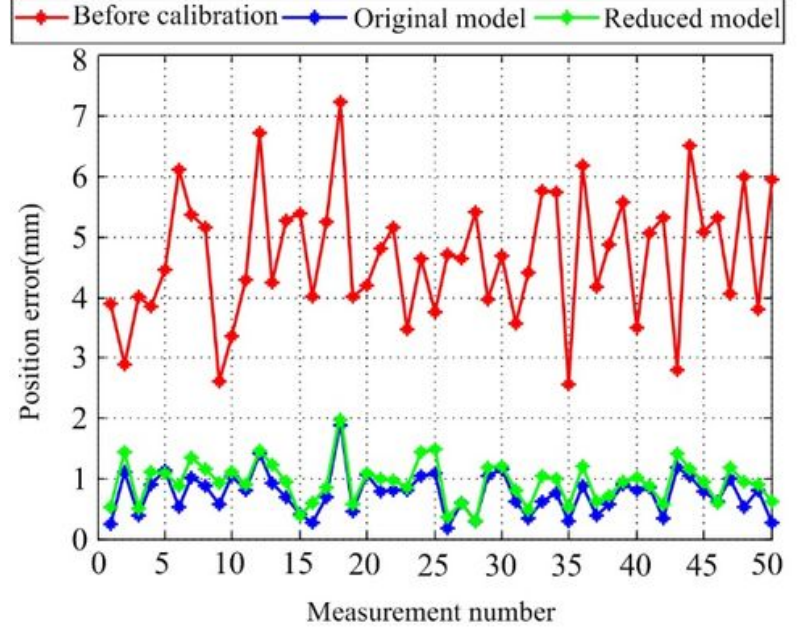

(a)

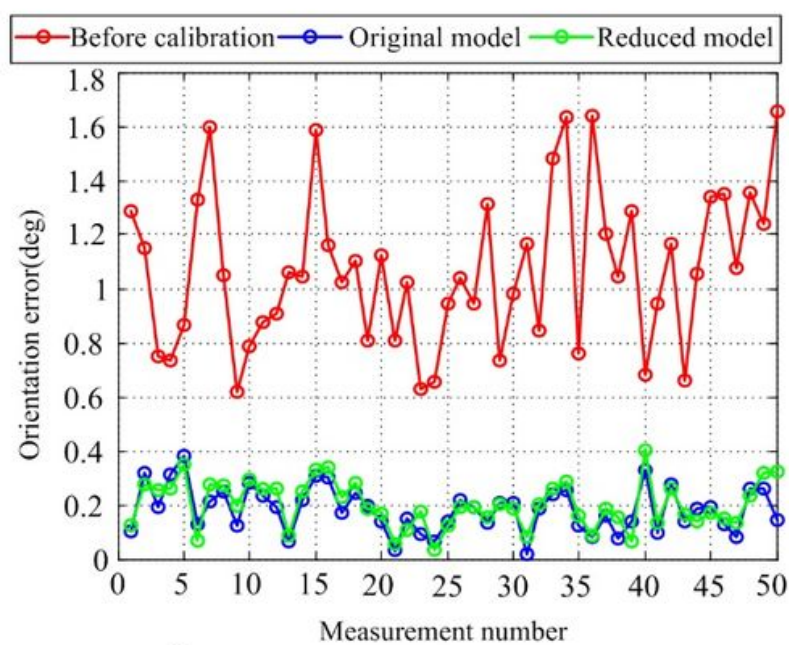

(b)

\section{Figure 5}

Pose errors of the robot before and after calibration (a) position error (b) orientation error 